\title{
Coronary thrombosis and platelet/fibrin microemboli in death associated with acute myocardial infarction
}

\author{
RICHARD J FRINK, PIERCE A ROONEY Jr, JAMES O TROWBRIDGE, \\ JAMES P ROSE* \\ From the ${ }^{\star}$ Department of Cardiovascular Research and the Department of Pathology, Mercy Hospital of \\ Sacramento, California, and the Division of Forensic Pathology, Sacramento County Coroner's Office
}

SUMMARY The frequency and clinical significance of platelet/fibrin microemboli in the micro-co circulation were investigated in 24 patients whose deaths (before and during hospital admission)? were associated with acute myocardial infarction. An acute coronary thrombus was present in all the hearts. In nine hearts an acute thrombus was found in more than one major epicardial coro-T nary artery. A total of 35 acute thrombi were found in the 24 hearts. Platelet/fibrin microembolio were found in $19(79 \%)$ hearts. Eighteen patients died in hospital. The hearts of 16 of these casesa showed microemboli; 16 had important arrhythmias or various forms of heart block; 13 showed acute pathological changes in the conduction system. Fourteen of the deaths in hospital were $e_{\infty}$ primarily the result of cardiogenic shock and four were primarily caused by arrhythmia. Six of the deaths that occurred before admission to hospital were regarded as being arrhythmic in origin. Three of these showed microemboli and the other three had acute pathological changes in the conduction system. Microemboli were found in two (24\%) of 12 control hearts.

Coronary thrombosis was found in most deaths caused by acute myocardial infarction ando platelet/fibrin microemboli were present in the majority of such hearts. These may arise from the coronary thrombus in the larger upstream vessel supplying the microcirculation.

Myocardial infarction is caused by or is closely associated with acute coronary thrombosis. Recent studies show that the frequency of occlusion in acute myocardial infarction decreases during the first 24 hours of a transmural infarction. ${ }^{1}$ These data suggest that lysis of thrombi and even of occlusions may occur in the course of acute infarction and may convert an occlusion into a non-occlusive thrombus. The exposed surface of a non-occlusive thrombus is often covered with friable, loosely attached platelet/fibrin aggregates. ${ }^{2}$ Some of these fragments undoubtedly pass distally to the microcirculation. ${ }^{34}$ The clinical significance of these microemboli has not been fully determined, but they have been reported in association with lethal rhythm disturbances, particularly ventricular arrhythmias,

Requests for reprints to Dr Richard J Frink, Heart Research Foundation of Sacramento, 3900 J Street, Sacramento, California 95819, USA.

Accepted for publication 13 August 1987 myocardial infarction, and sudden cardiac death. ${ }^{5-7}$ This report will show that many patients who die of acute myocardial infarction not only have acutecoronary thrombosis, but also have platelet/fibrin 3 . microemboli in the microcirculation, commonly dis-ô tal to an acute coronary thrombus. In addition, most of these patients have important arrhythmias, ando they often have acute pathological changes in the conduction system.

\section{Patients and methods}

The hearts of 24 patients ( 17 men and seven women aged 32-93 (mean 59)) who died of a microscopicallyo confirmed acute myocardial infarction were subjecto ted to a detailed pathological study of the coronary arteries, conduction system, and myocardium. Six ${ }^{\circ}$ patients died suddenly out of hospital and their hearts were obtained from the Sacramento County coroner's office. No electrocardiographic data were? available for these six patients, and their deaths are assumed to have been caused by arrhythmia. None 
of them had evidence of myocardial rupture or congestive changes in the lungs. Eighteen patients died after admission to the hospital and for all of them we had monitor strips or electrocardiograms showing the presence or absence of arrhythmias. Thirteen patients had been given anticoagulants, heparin, or warfarin.

Hearts were selected on the basis of (a) microscopic evidence of acute myocardial infarction; (b) satisfactory injection of the coronary arteries with a barium gelatin mass; $(c)$ absence of previous heart operation, coronary angioplasty, or streptokinase. No patients meeting these criteria were excluded. The cause of death in 14 hospital patients was cardiogenic shock; a lethal arrhythmia, confirmed electrocardiographically had caused the deaths of the other four hospital patients. The infarctions were classified as transmural (full thickness of the ventricular wall) or non-transmural.

We used a postmortem, angiographic, photographic technique with a coloured barium gelatin mass to study the hearts. ${ }^{2}$ Briefly, hearts were obtained fresh and uncut at the necropsy table, the coronary arteries were cannulated, and injected with a coloured barium gelatin mass. All hearts were injected within 24 hours of death.

After fixation in formalin, the entire length of all major epicardial coronary arteries was dissected from the heart and decalcified. $X$ rays were taken of the dissected coronary arteries before and after decalcification. Figure 1 shows a typical barium filled coronary artery. Arteries were then cut every 2-3 mm, embedded in paraffin and sectioned at $6 \mu \mathrm{m}$. These coronary artery sections were stained with haematoxylin eosin and also with Martius scarlet blue stain to detect fresh fibrin. Ten or more blocks of myocardium were taken from standardised sites and any suspected areas of infarction. Blocks of tissue containing the sinus and atrioventricular nodes, His bundle, and proximal bundle branches were removed and embedded for histological study. All sections of myocardium, atrioventricular node, and sinus node were stained with haematoxylin eosin and Martius scarlet blue. All microscopical sections were thoroughly searched for platelet/fibrin microemboli. The colouring material used in the injection mass identified the myocardial distribution of a given coronary artery. Thus fragments found in the distribution of a given coronary artery were assumed to have originated in or to have passed down through this artery.

An acute coronary thrombus in the main epicardial arteries was defined as an attached, organised mass of fibrin and platelets that stained appropriately with the Martius scarlet blue stain. Platelet/fibrin microemboli were identified micro-

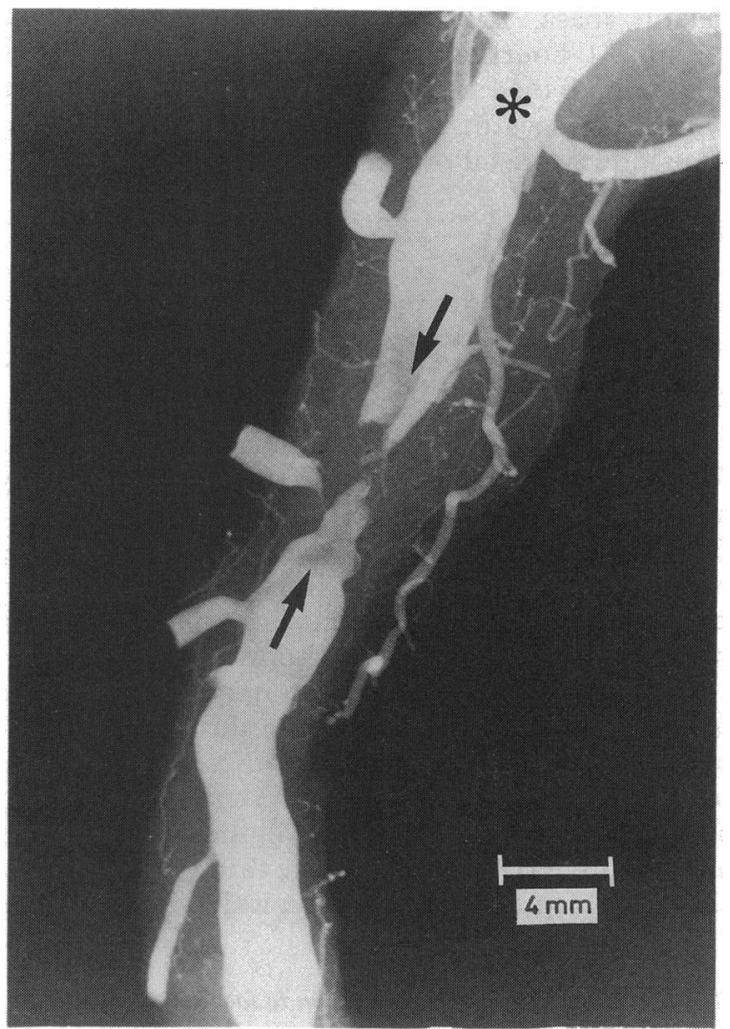

Fig 1 x ray taken of an epicardial right coronary artery after decalcification. Asterisk indicates area close to coronary ostium and arrows show the site of filling defects proximal and distal to the obstruction, which was confirmed to be an acute thrombus.

scopically as organised groups of platelets and fibrin, with clearly identifiable fibrin strands, present in a small myocardial arteriole. Platelet aggregates without fibrin were not counted in this study. Postmortem fibrin was distinguished as a looser, more filamentous structure, with no organisation of the fibrin.

\section{CONTROL PATIENTS}

The hearts from 12 men (aged 19-81, mean 36) were examined to determine the frequency of microemboli in patients whose deaths were caused by noncardiac disease, accidents, or violence $(n=10)$ and also those whose deaths were cardiogenic but noncoronary in origin ( $n=2$; myocarditis, hypertrophic subaortic stenosis). Of the 10 patients dying of noncardiac disease, accidents, or violence, six hearts were injected in a manner identical to that described above and four were processed and examined without the use of injection material. The two cardiogenic control hearts were injected with the barium 
gelatin mass. All control hearts were prepared within 24 hours of death. Three patients, all of whom had died of non-cardiogenic causes, died within six days of admission to the hospital. The remainder were all out-of-hospital deaths.

\section{Results}

The table shows the pathological findings in all 24 cases, subdivided according to transmural or nontransmural infarction. The term "global infarction" indicates that acute infarction was found throughout the myocardium. An acute coronary thrombus was found in all the hearts. These thrombi were firmly attached to the arterial wall and had irregular surfaces with many loosely attached fragments. In nine hearts an acute coronary thrombus was found in more than one epicardial coronary artery. In the 24 patients, 35 acute coronary thrombi were found in separate major epicardial coronary arteries. The infarction was distal to an acute thrombus in each heart, with the exception of those with global infarction. Microemboli were present in $19(79 \%)$ of all hearts, and most were found in the distribution of an artery that contained an acute thrombus. Figure 2 shows the appearance of a typical microembolus.
Microemboli were also found in three patients with left ventricular mural thrombi. In two of these. patients, microemboli were found in the distribution of an artery that did not contain an acute coronary thrombus.

Although all hearts contained a coronary throm $\frac{\overline{\bar{c}}}{\frac{\bar{c}}{2}}$ bus, emboli were more common in patients with transmural infarction than in patients with none transmural infarction. The difference, however, was not significant $(p>0 \cdot 10)$. Five of the six patients with non-transmural infarction died outside hospital: three had microemboli, and four had pathological changes in the conduction system.

Eighteen deaths occurred after admission to the hospital. All died within 17 days of admission; eight within the first 48 hours. There were significani arrhythmias, over and above single atrial or ventrico ular unifocal extrasystoles in 16 patients; five of these also developed various forms of new onset heart block. The forms of heart block included $2: \mathrm{h}^{\mathrm{T}}$ block, right bundle branch block, left bundle brancle block, atrioventricular dissociation, and junctiona rhythm. There was ventricular tachycardia or ventricular fibrillation (other than as an agonal rhythm $\overrightarrow{8}$ in $12(60 \%)$ patients. Two patients did not show anyo type of arrhythmia. The conduction system was

Table Site of thrombus in relation to location of infarction and distal microemboli in patients with transmural and non-transmural infarction

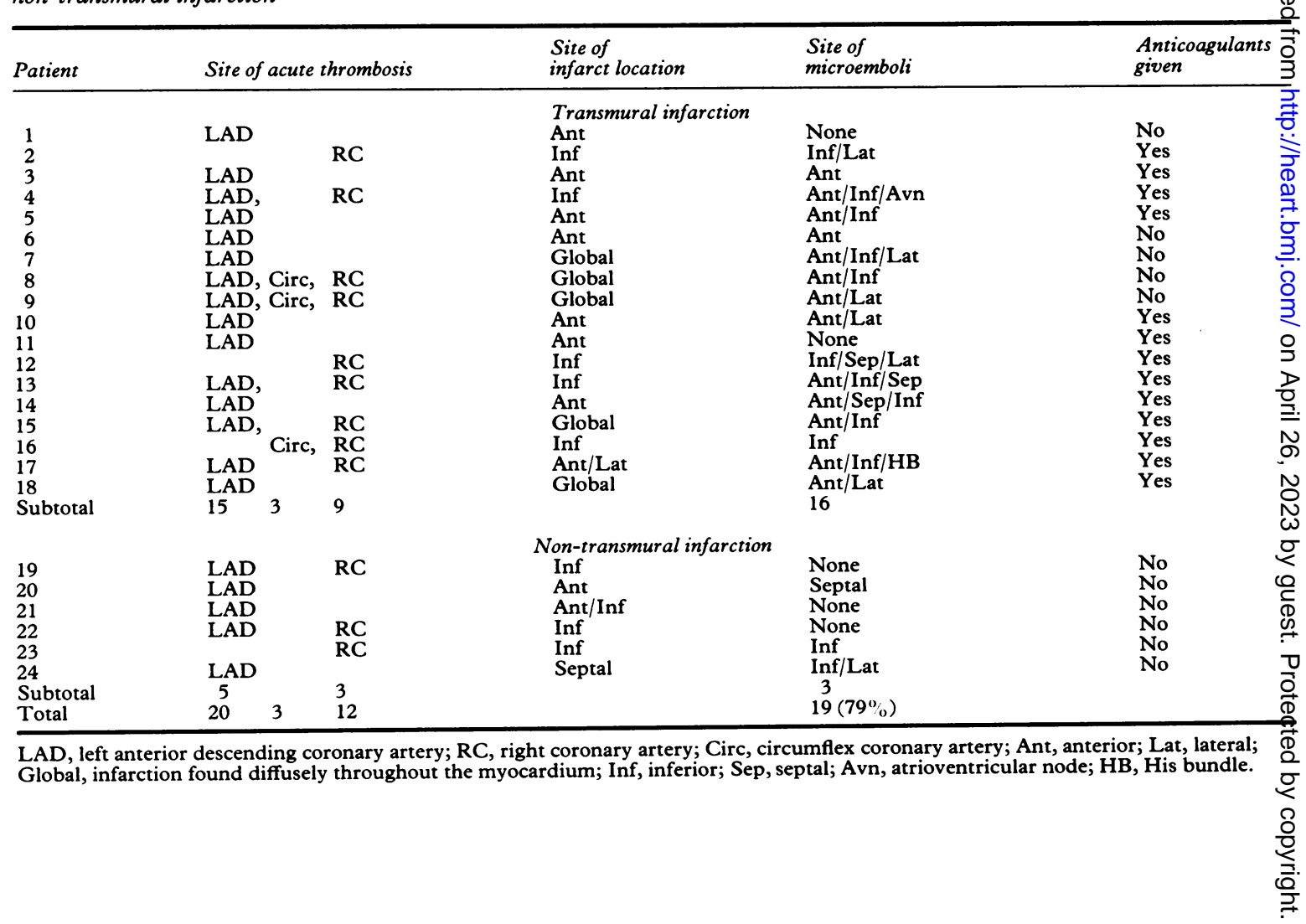




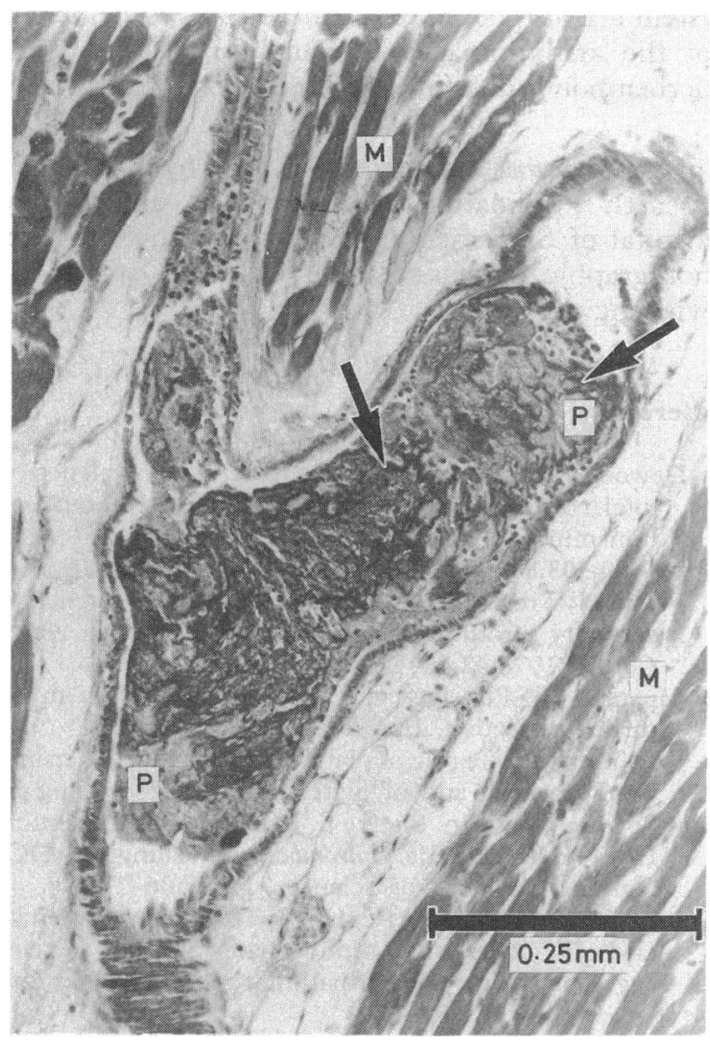

Fig 2 Photomicrograph showing small intramyocardial arteriole containing a microembolus (black arrow).

$M$, myocardium; $P$, platelets. Solid black arrows show fibrin strands forming islands of platelets.

abnormal in $13(72 \%)$ patients. The crux of the heart was supplied by the right coronary artery in 23 hearts.

Of 13 patients on anticoagulant treatment, 11 were considered to be fully anticoagulated, and of these, 10 showed microemboli.

\section{CONTROL STUDIES}

Coronary thrombosis was not found in any of the control hearts and there was no evidence of coronary disease in any of them. Two patients showed a single, but well defined, platelet/fibrin microembolus in the myocardial microcirculation. These two patients had been admitted to hospital for three and six days before death, both had severe intracranial haemorrhage, and both hearts were injected with the barium gelatin mass. No microemboli were found in any of the other control patients, whether or not they had been injected with the barium gelatin mass. This indicates that the use of the injection mass does not affect the detectability of the microemboli. The conduction system was examined in all control hearts and no acute changes were found.

\section{Discussion}

\section{PLATELET/FIBRIN MICROEMBOLI IN THE MICROCIRCULATION}

We found platelet/fibrin microemboli in most patients who died of acute myocardial infarction, especially of transmural infarction. We found that the more comprehensive the search for microemboli, the more we found. There are three possible sources of the platelet/fibrin microemboli and these are not necessarily mutually exclusive. First, the formation of microemboli may be an agonal event or may occur soon after death. Platelet aggregation during acute illness has been reported $^{8}$; for this reason, pure platelet aggregations were not counted in this study. The fragments we considered to have been formed before death were tightly knit, compact, wellformed fragments of fibrin, with or without platelets (fig 2), unlike the loose, unorganised appearance of postmortem fibrin.

A second possibility is that microemboli may occur as a result of increased platelet reactivity, which is sometimes seen in patients with acute myocardial infarction. ${ }^{9}$ The condition is, as yet, poorly defined, and there are no pathological studies for comparison. Except in the two cases of intracranial haemorrhage, however, microemboli were only found in association with a thrombus. Thus it is unlikely that they represent a primary event.

The third possibility is that the microemboli may originate from an acute coronary thrombus located in the upstream coronary artery. Most microemboli in this study were distal to a coronary thrombus, which could have served as a source for these fragments. In addition, those patients with ventricular mural thrombi had microemboli in the microcirculation in the distribution of an artery that did not contain a thrombus. Furthermore, in a previous study of coronary thrombosis and sudden death, ${ }^{2}$ platelet/fibrin microemboli were found in most cases. The thrombi in this and the current study were characterised by their irregular surface and loosely attached fragments. Clearly, the thrombus is in a dynamic state of change undergoing episodic formation and dissolution as a result of the flow of blood, platelet disaggregation, and fibrinolysis. ${ }^{14} 10$ The body of evidence thus supports the notion that platelet/fibrin microemboli originate from a proximal thrombus.

Microemboli were found in the microcirculation irrespective of the length of hospital stay. But neither the time of embolisation nor the state of the 
fibrinolytic system at the time of death were known. None the less, as all patients died as a result of a myocardial infarction and the majority of the patients had microemboli, it is possible that platelet/fibrin microemboli contributed to the haemodynamic and/or electical instability in these patients. The present data show that such embolisation is a possibility irrespective of the age of the infarction.

Anticoagulation seemed to have little effect on the presence or absence of microemboli. The effectiveness, therefore, of anticoagulants to retard the thrombotic process and reduce the frequency of microemboli is questionable.

CLINICAL MANIFESTATIONS OF MICROEMBOLI An important question is the relation between microemboli and death associated with acute myocardial infarction. Numerous investigators have provided evidence that microemboli may be involved in focal fibrosis, ${ }^{11}$ subendocardial injury, ${ }^{12}$ unstable angina pectoris, ${ }^{13}$ and sudden cardiac death. ${ }^{267}$ To our knowledge, however, no correlation between microemboli and death associated with acute myocardial infarction has yet been made. The present finding of a high frequency of microemboli indicates that the two might be related. The above evidence also suggests that microemboli do have pathogenetic potential and that they are apparently capable of causing various clinical symptoms.

The relation between microemboli and arrhythmias is not clear. Important arrhythmias or recent onset heart block, assuming that all out-of-hospital deaths were arrhythmic in origin, were present in $92 \%$ of patients in this study. Microemboli were found in $79 \%$. Most (61\%) arrhythmias were ventricular in origin. Conduction system abnormality was also present in $61 \%$ of cases. The occurrence of arrhythmias, therefore, correlated reasonably well with the presence of microemboli, as well as with some types of conduction system abnormalities. The pathological findings, however, could not be correlated with specific arrhythmias. In several cases microemboli were found in or immediately adjacent to the conduction system. This evidence does not prove that microemboli caused the arrhythmias or heart block, but neither does it rule out that possibility. At the very least microemboli should be considered a possible mechanism for producing transient, severe, reversible ischaemia of focal areas of the myocardium and/or the conduction system.

In summary, platelet/fibrin microemboli in the microcirculation are common in patients dying of acute myocardial infarction and they seem to be derived from a proximal thrombus. Arrhythmias and acute pathological changes in the conduction system are also common in these cases; whether or not the arrhythmias are related to platelet/fibrin microemboli is unknown and requires further studyo

This study was supported in part by the Hearis Research Foundation of Sacramento and Mercy⿳a Hospital of Sacramento. We thank Ken Shiba forn photographic assistance and Dr Louis Ostrach forreviewing the article.

\section{References}

1 Dewood MA, Spores J, Notske R, et al. Prevalence of total coronary occlusion during the early hours of transmural myocardial infarction. $N$ Engl $J$ Medo 1980;303:897-902.

2 Frink RJ, Trowbridge JO, Rooney PA. Nonobstructive coronary thrombosis in sudden cardiac death. $\mathrm{Am} \Gamma$ Cardiol 1978;42:48-51.

3 Mustard JF. Platelets and thrombosis in acute myocardial infarction. Hosp Pract 1972;7:115-28.

4 Jorgensen $\mathrm{L}$. The role of platelet embolism from crumbling thrombi and of platelet aggregates arising in $\overrightarrow{0}$ flowing blood. In: Sherry S, Brinkhous KM, Gentonoळ E, Stengle JM, eds. Thrombosis. Washington, DC: National Academy of Sciences, 1969:506-33.

5 Jorgensen L, Rowsell HC, Hovig T, Glynn MF, Mustard JF. Adenosine diphosphate-induced platelet aggregation and myocardial infarction in swine. Lab Invest 1967;17:616-44.

6 El-Maraghi N, Genton E. The relevance of platelet and fibrin thromboembolism of the coronary microcirculation, with special reference to sudden cardiac death. Circulation 1980;62:936-44.

7 Davies MJ, Thomas AC, Knapman PA, Hangartner JR. Intramyocardial platelet aggregation in patients with unstable angina suffering sudden ischemion cardiac death. Circulation 1986;73:418-27.

8 Hampton JR, Mitchell JRA. Abnormalities in platelet behaviour in acute illnesses. $\mathrm{Br}$ Med $J$ 1966;i: 1078-80.

9 Mehta P, Mehta J. Platelet function studies in coronary artery disease. V. Evidence of enhanced platele $\varrho$ microthrombus formation activity in acute myo cardial infarction. Am J Cardiol 1979;43:757-60.

10 Kinlough-Rathbone RL, Packham MA, Mustard JF Vessel injury, platelet adherence, and platelet sur-o vival. Arteriosclerosis 1983;3:529-46.

11 Mills NL, Ochsner JL. Distal thromboembolism anç proximal coronary arteriosclerotic lesions. Surgery 1972;72:1030-6.

12 Moore S, Belbeck LW, Evans G, Pineau S. Effects o@ complete or partial occlusion of a coronary artery Lab Invest 1981;44:151-7.

13 Falk E. Unstable angina with fatal outcome: dynamico coronary thrombosis leading to infarction and/o sudden death. Autopsy evidence of recurrent muraD thrombosis with peripheral embolization culmin $\frac{\rho^{+}}{\mathrm{D}^{+}}$ ating in total vascular occlusion. Circulation 1985;71음 699-708. 